\title{
Materials and process discovery by correlated STEM imaging and spectroscopy with electrical testing
}

Andrew Wagner ${ }^{1}$, John Nugent ${ }^{2}$ and Kumar Virwani ${ }^{2}$

${ }^{1}$ Intel Corporation, Beaverton, Oregon, United States, ${ }^{2}$ Intel Corporation, United States

The need for high-volume transmission electron microscopy (TEM) has increased exponentially in semiconductor technology development(TD). Aggressive scaling and complexity require accuracy and precision that is better than conventionally possible and with reduced human input. Rule-based image processing and machine learning have proven useful for increased volume of measurements, however there are limits to objective determination of resolution and confidence, particularly for machine learning-based approaches which themselves rely on some ground truth. Any new metrology tool must be benchmarked against best known methods.

Spatial calibration of TEMs, for example, is often performed imaging a crystalline material with known lattice constant. Spatial resolution is often demonstrated by analyzing the Fourier transform of a similar image. Metrology resolution, particularly for typical semiconductor device specimens, requires additional care. Rough interfaces, contrast variations, thick specimens, and the method of measurement itself all contribute to the accuracy, precision, and bias of a measurement.

Dimensional metrology is often performed by hand, however the person-to-person variation is often larger than the process tolerance. Rule-based image processing can improve consistency, however development of these rules is time consuming and they remain sensitive to variation in imaging and process conditions. More recently machine learning and particularly convolutional neural networks (CNNs) have been deployed, however they require training and the nature of convolution can lead to results that are not sufficiently accurate or precise for defining interfaces [1]. Horwath et al make a convincing argument that the simplest models are often the most beneficial. When deploying machine learning to materials science, the goal is often to accurately reproduce a known process or to learn something new. To satisfy these, physical constraints can aid in interpretability and performance [2]. Whether measurements are performed by hand, via rule-based processing, or incorporate machine learning the results must be evaluated for quality.

Here we provide an example benchmark for new metrology that measures a well-defined physical phenomenon. A series of structures are electrically programmed, effecting a known volumetric change. For the length scales at play, the volumetric change leads to a linear change of about half an atom. This is a convenient benchmark for metrology resolution as devices today have process marginality requirements that aggressively push this level of tolerance. 
To minimize the effects of within-wafer variation, a series of neighboring structures are programmed in a defined, but random pattern of ' $S$ ' and ' $R$ ' states. The same structures are carefully prepared for cross-sectional TEM imaging and then imaged, measured, and assigned to their respective state. The population of ' $S$ ' and ' $R$ ' states from a series of samples, Figure 1, show a clear separation. An analysis of their means confirms that there is a statistically meaningful shift in their distributions, which also correlates with the expected change in cross-sectional area. The area measurements effectively consist of many hundreds of individually identified endpoints. Looking only at individual measurements, for example the mid width of the structure, shows a similar distribution. Interestingly, the change in width found is significantly larger than expected and deviates from the observed cross-sectional area. The measured heights showed no statistical difference between the states. Put in context, this is to be expected. Due to the aspect ratio of the structures and their confinement within surrounding material, there exists a compressive stress which restricts the ability to expand vertically and most volume change constrained to the horizontal plane.

This method provides an indirect, but physics-based ground truth against which any new metrology can be benchmarked. The process of record prior to the method implemented here was not able to show a statistical difference when measured on the same micrographs. Indeed, an analysis of variance suggests a significantly different contribution of the volumetric change for the superior metrology compared to the process of record. Similar approaches can be implemented for spectroscopic mapping of well-defined nanoscale structures.

With confidence established, we will show how large metrology data sets can be directly correlated to electrical parametric testing, Figure 2. During the early stages of process development, within-wafer process variation is naturally large and provides a rich dimensional space for materials and process discovery. Commonality analysis of these large datasets can identify superior structures and the characteristic traits which deliver these results. This is only possible when generating large and highly correlated data sets containing electrical parametrics and respective dimensional and compositional information. Such discovery can make highly impactful contributions to technology development, reducing the number of process turns and cycle times. As image processing, metrology, chemical analysis and fusion of data advance, there exists a virtuous cycle that will support greater innovation in the materials and data sciences. 
(a)

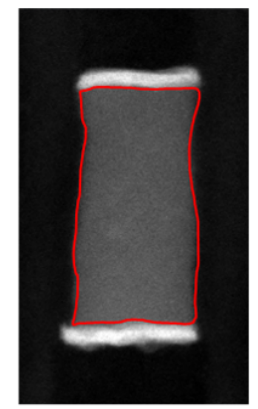

(b)

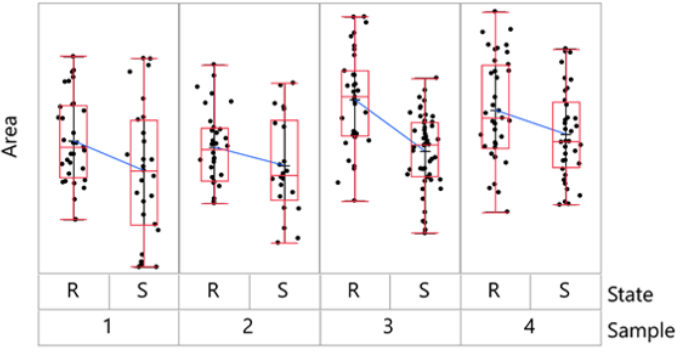

(d)

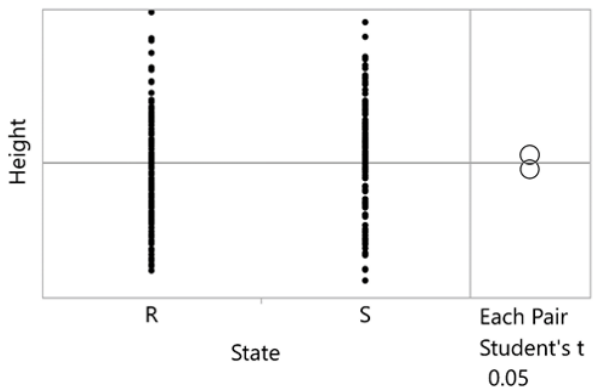

(c)

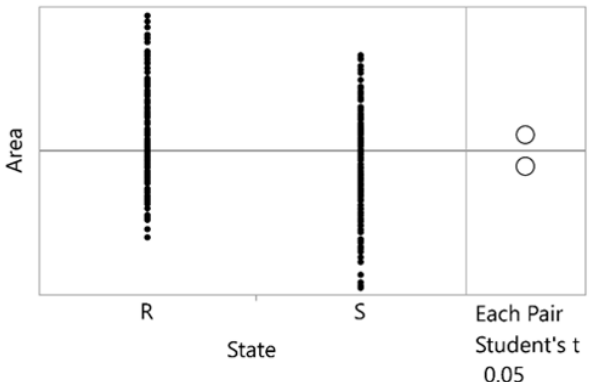

(e)

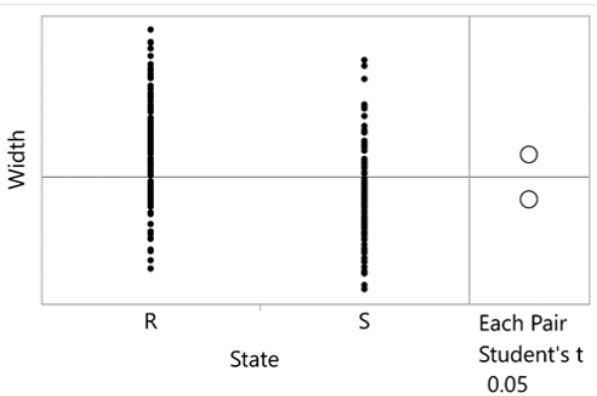

Figure 1. Figure 1. (a) STEM micrograph of sample. (b) A comparison of the measured areas of the two states, showing a statistical separation between populations matching that expected. (c) Means comparison showing statistical separation between states. (d) Means comparison of specimen heights showing no statistical separation. (e) Means comparison showing statistical separation of widths.

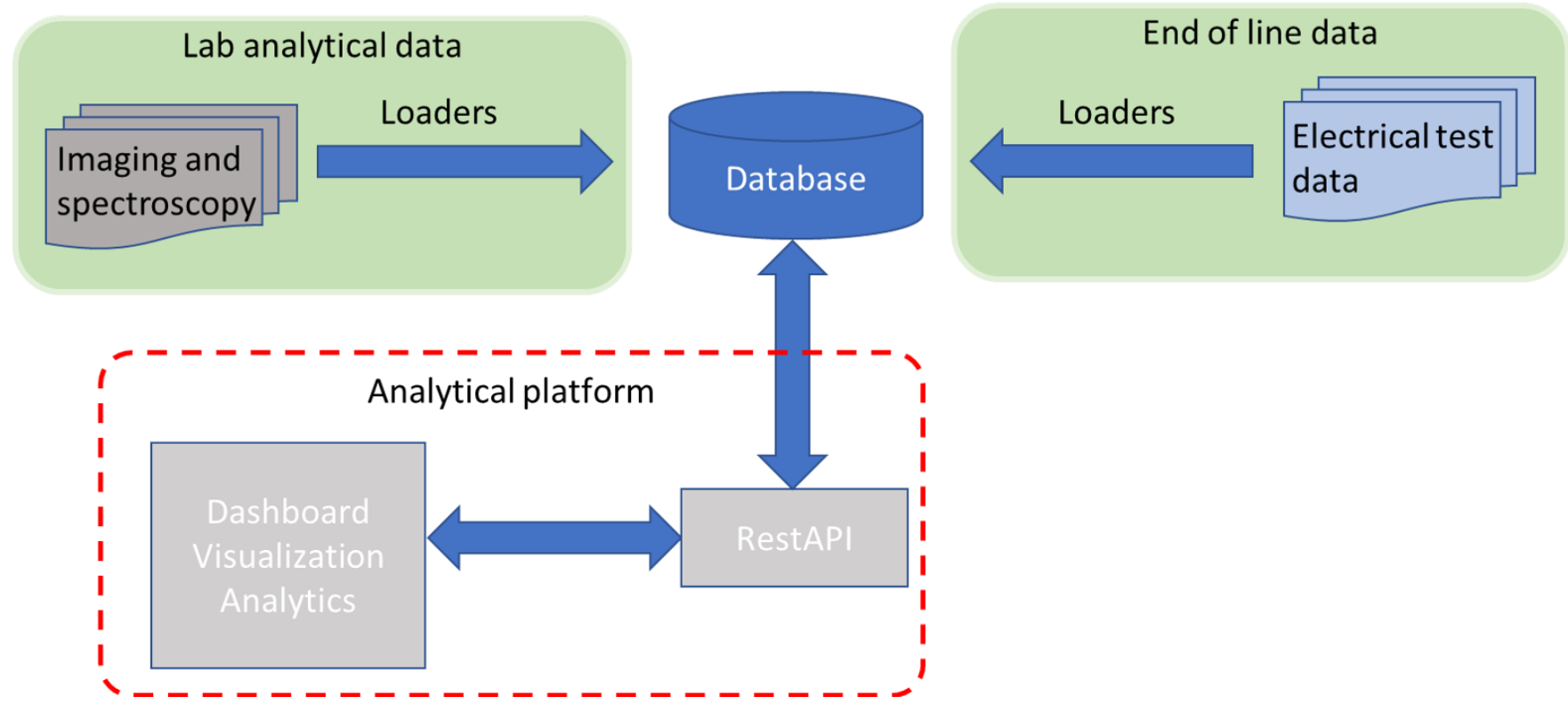

Figure 2. Figure 2. High-level schematic of system for multivariate analysis of device structure, composition and electrical performance. With proper design and modularization, analytics can be continuously added and optimized.

\section{References}

[1] J. Horwath, D. Zakharov, R. Megret and E. Stach, "Understanding important features of deep learning models for segmentation of high-resolution transmission electron microscopy images," npjComput Mater, pp. 6, 108, 2020. 
[2] R. Vasudevan, M. Ziatdinov, L. Vlcek and S. Kalinin, "Off-the-shelf deep learning is not enough, and requires parsimony, Bayesianity, and causality," npjComput Mater, pp. 7, 16, 20 